\title{
Diffraction Efficiency of Volumetric Magnetic Holograms with Magnetophotonic Crystals
}

\author{
Ryosuke Isogai, Naoto Sagara, Taichi Goto, Yuichi Nakamura, Pang Boey Lim, and Mitsuteru Inoue \\ Toyohashi University of Technology, 1-1 Hibarigaoka, Tempaku, Toyohashi, Aichi 441-8580, Japan
}

Hologram memory is a promising data storage technology with a high recording density and fast data-transfer rate. Magnetic garnet media such as $\mathrm{Bi}_{1.3} \mathrm{Dy}_{0.85} \mathrm{Y}_{0.85} \mathrm{Fe}_{3.8} \mathrm{Al}_{1.2} \mathrm{O}_{12}$ (substituted rare-earth iron garnet, SRIG) films have advantages of rewritability and unnecessity of shielding. The diffraction efficiency of these garnet films, however, is not sufficient to apply to storage devices. In this paper, we proposed a use of magnetophotonic crystals (MPC) as hologram media and calculated their diffraction efficiency. The results indicated that MPC media showed a high diffraction efficiency compared with single SRIG films, and especially the MPC medium with the structure of substituted gadolinium gallium garnet substrate / $\left(\mathrm{Ta}_{2} \mathrm{O}_{5} / \mathrm{SiO}_{2}\right)^{2} / \mathrm{SRIG} /\left(\mathrm{SiO}_{2} / \mathrm{Ta}_{2} \mathrm{O}_{5}\right)^{2}$ with the SRIG thickness of $3.88 \mu \mathrm{m}$ exhibited the diffraction efficiency of $0.36 \%$. A large Faraday rotation angle and deep magnetic hologram fringe originated from the localization of light results in such a high diffraction efficiency.

Key words: magnetic hologram, magnetophotonic crystal, diffraction efficiency, magneto-optical recording, thin film

\section{Introduction}

Hologram memory is a promising data storage technology with a high recording density and fast datatransfer rate ${ }^{1)}$. Photopolymer is mainly used as writeonce hologram media because they have a high diffraction efficiency. As a candidate for rewritable hologram media, magnetic films such as transparent magnetic garnet films have been studied ${ }^{2)}$.

Magnetic holograms written by thermomagnetic method into a magnetic film can be reconstructed with magneto-optical (MO) effect ${ }^{3}$. One of the advantages of magnetic holograms is the high tolerance to the noise caused by stray light, because the polarization plane of the diffracted light rotates $90 \mathrm{deg}$ and it enables the isolation between the signal and noise (non-rotated stray light) with a light polarizer. However, the light transmitted through the magnetic medium has the slightly rotated component and generates a leakage light through the polarizer or the noise. To describe both the efficiency of diffraction and amount of this noise with just one index, we have defined the ratio of a first-order diffracted beam intensity to a zero-order transmitted beam intensity as a diffraction efficiency for magnetic holograms ${ }^{4)}$. A transparent magnetic garnet, $\mathrm{Bi}_{1.3} \mathrm{Dy}_{0.85} \mathrm{Y}_{0.85} \mathrm{Fe}_{3.8} \mathrm{Al}_{1.2} \mathrm{O}_{12}$ (substituted rare-earth iron garnet, SRIG) is a well-known MO material with a large MO effect ${ }^{5}$. The diffraction efficiency of a single SRIG film is, however, not sufficient to apply to storage devices so far.

To improve the diffraction efficiency, a use of magnetophotonic crystal (MPC) media, where a magnetic layer sandwiched between two Bragg mirrors, seems effective $^{6)-8)}$. In a MPC structure, an incident light is localized in the magnetic defect layer by multipath reflection, and it enhances the MO effect and provides a large Faraday rotation angle. The improvement of the diffraction efficiency is expected by increasing rotation angle with a MPC structure. In this paper, we investigated the diffraction efficiency of MPC hologram media by calculation and clarified the mechanism of a high diffraction efficiency with MPC media.

\section{Magnetic hologram}

\subsection{Writing method}

In magnetic holograms, an interference pattern is recorded into a magnetic film as magnetization directions by thermomagnetic method. Two incident beams, named signal beam and reference beam, were radiated in a medium magnetized perpendicularly in a single direction, and the temperature distribution associated with the hologram is formed by optical absorption and heating. As a result, the magnetization in the region where the temperature exceeds the Curie temperature was lost and reversed (e.g. by the stray magnetic field) during the cooling process to form the magnetic hologram fringe.

\subsection{Reconstruction method}

To obtain the reconstructed image, only the reference beam is radiated to the medium. When the light propagates through a magnetized material, the Faraday effect occurs. The degree of polarization rotation angle of the light is determined by the material-specific Faraday coefficient and the thickness of the medium, and the rotation direction is dependent on the magnetization in the domain through which the light passes. The light transmitted a magnetic diffraction grating like a magnetic hologram has the spatially distributed component of the polarization plane ( $x$ axis in Fig. 1) and the $x$ components in the opposite directions mean opposite in phase; therefore, a magnetic hologram is considered as a phase hologram written magnetically. 


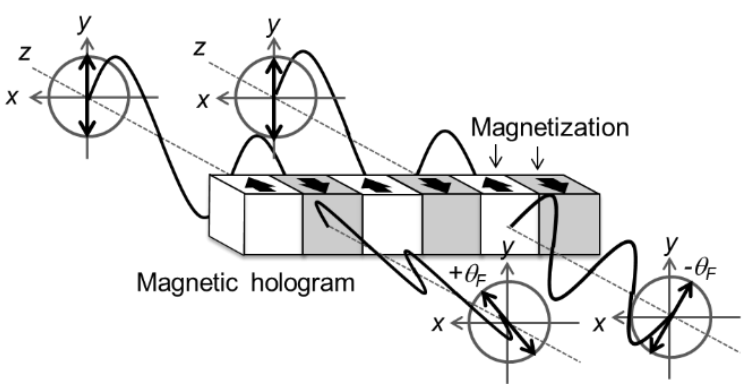

Fig. 1 Reconstruction mechanism of magnetic hologram

Since the magnitude of the $x$ components depends on the rotation angle of the polarization plane, a magnetic media with a large MO effect brings a bright reconstructed beam, namely high diffraction efficiency.

\section{Calculation method}

The structure of the MPC media used for the simulations was substituted gadolinium gallium garnet substrate / $\left(\mathrm{Ta}_{2} \mathrm{O}_{5} / \mathrm{SiO}_{2}\right)^{r} / \mathrm{SRIG} /\left(\mathrm{SiO}_{2} / \mathrm{Ta}_{2} \mathrm{O}_{5}\right)^{r}$, where $r$ is the number of repeated pairs in the Bragg mirrors. The thickness of the $\mathrm{SiO}_{2}$ and $\mathrm{Ta}_{2} \mathrm{O}_{5}$ layers are determined as $\lambda / 4 n$, where $\lambda$ is the resonant wavelength of $532 \mathrm{~nm}$ and $n$ is the refractive index of each material. The material parameters used in the simulations were experimentally determined and are shown in Table 1. The thickness of the SRIG film, the number of repeated pairs in the Bragg mirrors, and the writing power density were varied.

The diffraction efficiencies of the SRIG single films and MPC structures were calculated by finite element method (COMSOL Multiphysics 4.3a). In the simulations, two-beam interferometer system is used to write and reconstruct a hologram. Two incident Gaussian beams $(\lambda$ $=532 \mathrm{~nm}$ ) with the diameter of $51.2 \mu \mathrm{m}$ were radiated in the medium for 50 psec. The beams were tilted $23^{\circ}$ from the normal direction of the surface of medium so that holograms with the spatial frequency of 1500 line pair/mm will be formed. The electric field strength distribution of the interfering light in the medium was computed to get the energy distribution absorbed by SRIG, and the time development of temperature profile was calculated. From this profile, the magnetic hologram fringes were determined, assuming that the magnetization in the region where the temperature exceeds the experimentally obtained Curie temperature of SRIG films of $150^{\circ} \mathrm{C}$ is reversed. Then the reference beam was radiated to the medium to reconstruct the signal beam. The diffraction efficiency $\eta$ was derived from the simulation results by the following equation ${ }^{4)}$,

$$
\eta=\frac{I_{1}}{I_{0}} \times 100(\%)
$$

where $I_{1}$ and $I_{0}$ are the intensity of the reconstructed first-order beam and that of the transmitted zero-order beam, respectively.
Table 1 Material parameters in the simulations ${ }^{\mathrm{a}}$

\begin{tabular}{cccc}
\hline Material & $n$ & $\kappa$ & $\theta_{\mathrm{F}}(\mathrm{deg} / \mu \mathrm{m})$ \\
\hline $\mathrm{SRIG}$ & 2.09 & 0.0186 & 2.26 \\
$\mathrm{SiO}_{2}$ & 1.45 & 0 & - \\
$\mathrm{Ta}_{2} \mathrm{O}_{5}$ & 2.16 & 0 & - \\
\hline
\end{tabular}

aRefractive index $n$, extinction coefficient $\kappa$, and specific Faraday rotation angle $\theta_{\mathrm{F}}$.

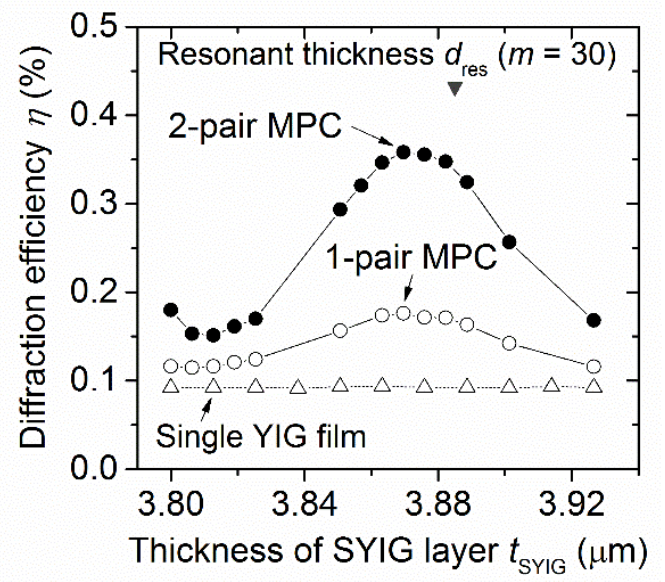

Fig. 2 The SRIG thickness dependence of the diffraction efficiency. The SRIG thickness of $3.876 \mu \mathrm{m}$ corresponds to the resonant thickness with $m=30$. In the MPC media, the constructive interference by Fabry-Pérot resonations causes oscillations in the thickness dependencies on the diffraction efficiency.

\section{Results and Discussion}

Fig. 2 shows the dependence of diffraction efficiency of the media on the SRIG thickness between 3.8-3.9 $\mu \mathrm{m}$, and this range of thickness is enough thick to provide the saturated diffraction efficiency with a SRIG single film ${ }^{9)}$. The writing power density, which is the total power density of the reference and signal beams, was chosen to obtain the maximum diffraction efficiency. The maximum diffraction efficiencies were obtained at the power densities of incident beams of 1100, 1700, and $2300 \mathrm{~J} / \mathrm{m}^{2}$ for the single film, 1-pair MPC, and 2-pair MPC structure, respectively. This increasing of the required incident energy is due to reflections at the surface of Bragg mirrors, and it results that the power density increased with increasing the number of Bragg mirror layers. In MPC structures, there are oscillations with a period of sub-wavelength in the thickness dependencies of the diffraction efficiency because of Fabry-Pérot resonance. The obvious maximum peak exists in Fig. 2 and its thickness is in good agreement with the resonant thickness $d_{\text {res }}$, given by Fabry-Pérot resonant condition for constructive interference between the Bragg mirrors,

$$
d_{r e s}=m \lambda / 2 n \cos \varphi,
$$

where $m$ is an integer, $\lambda$ is the wavelength of light, $n$ is the refractive index of SRIG, and $\varphi$ is the angle between the beam and the normal to the surface in the SRIG layer. 

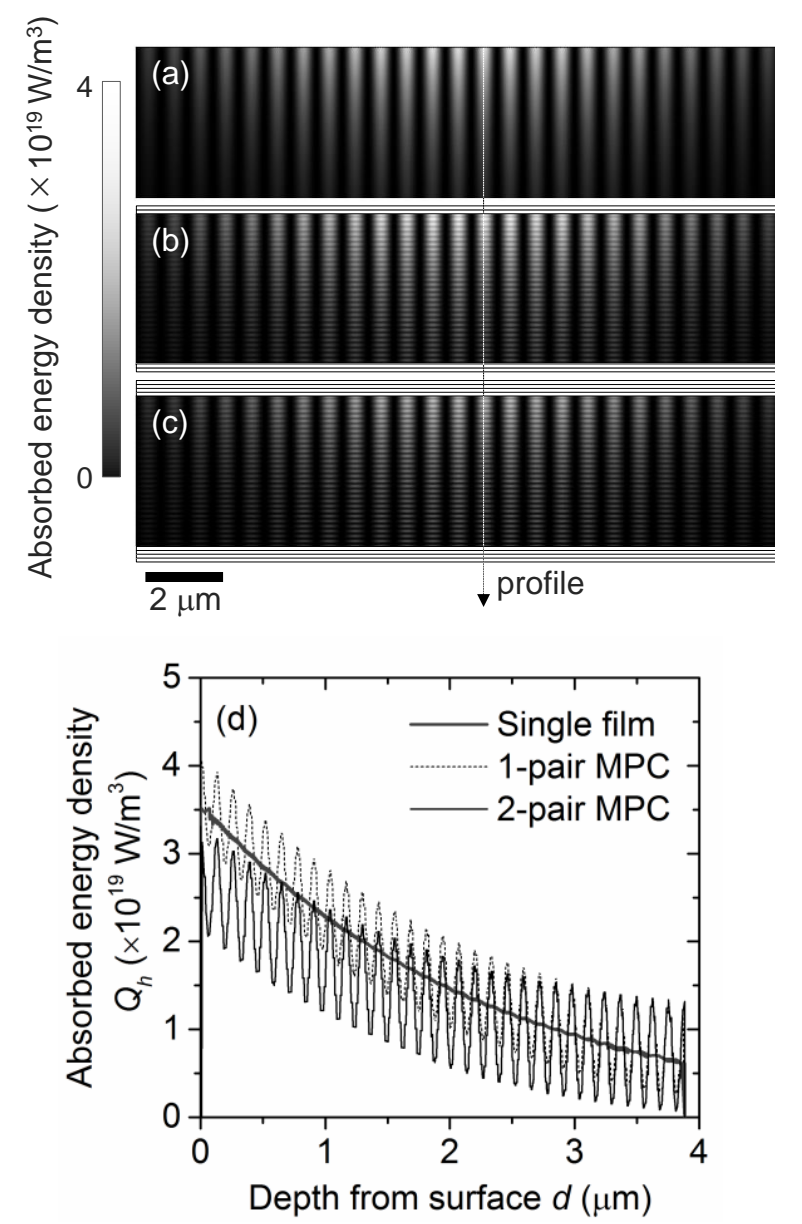

Fig. 3 Distribution of the absorbed energy density in the SRIG layer with the thickness of $3.88 \mu \mathrm{m}$ for the single SRIG film (a), 1-pair MPC (b), and 2-pair MPC (c). The profiles of the absorbed energy density along the line at the horizontal center of the fringes shown in (a)-(c) are shown in $(d)$.

The angle of the beam changes from the original incident angle in air of $23^{\circ}$ to $11^{\circ}$ because the refraction at the interface of the SRIG layer, then we set $\varphi$ to be $11^{\circ}$. The maximum diffraction efficiency of the single film and 1pair MPC were respectively $0.09 \%$ and $0.18 \%$. The high diffraction efficiency of $0.36 \%$, which was 4 times larger than that of the single film, was obtained for the 2-pair MPC with the SRIG thickness of $3.876 \mu \mathrm{m}$ (for the case of $m=30$ ). Similarly, when $m$ is another integer, the maximum diffraction efficiency is also present at the corresponding a resonant thickness. The results show that the localization of light at the resonant wavelength significantly affect the diffraction efficiency.

The diffraction efficiency of volumetric holograms are theoretically determined by the rotation angle from the magnetic fringe, hereafter simply referred to as fringe rotation angle $\theta_{\text {ir }}$, given by Eq. (3),

$$
\theta_{f r}=d_{w} \times \theta_{F}(\mathrm{deg}),
$$

where $d_{\mathrm{w}}$ is the depth of the magnetic fringe and $\theta_{\mathrm{F}}$ is the
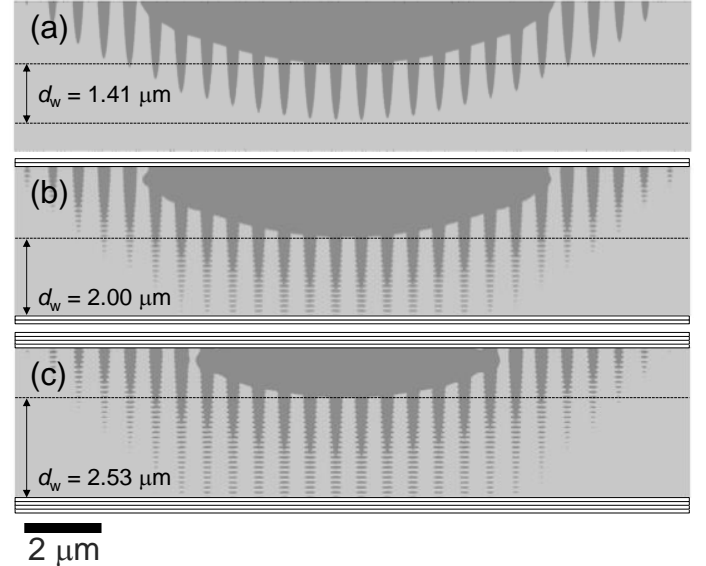

Fig. 4 Magnetic hologram fringes written in the SRIG layer with the thickness of $3.88 \mu \mathrm{m}$ for the single film (a), 1-pair MPC (b), and 2-pair MPC (c).

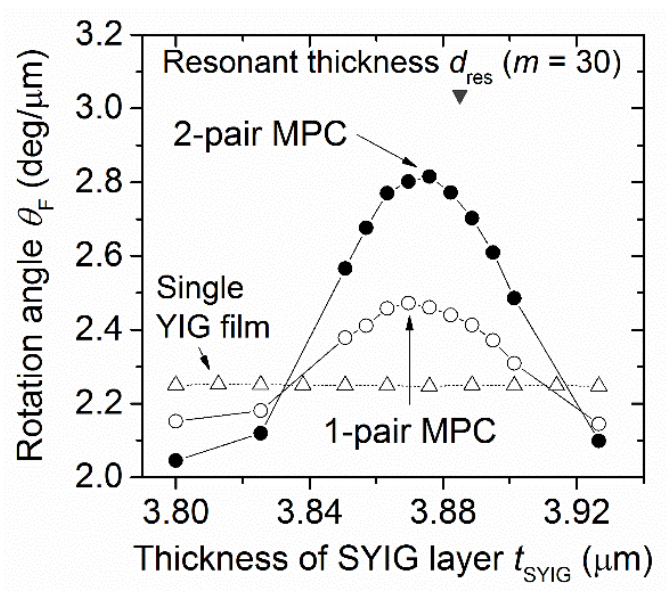

Fig. 5 Specific Faraday rotation angle of the magnetic hologram media. The enhancement of the rotation angle is observed at the resonant thickness due to the Fabry-Pérot resonation.

rotation angle per unit of thickness. When the width of the hologram fringes is constant, a hologram with a deeper fringe depth $d_{\mathrm{w}}$ provides a high diffraction efficiency.

To investigate the mechanism of the improvement of diffraction efficiency, the calculated absorbed energy density distribution in the media is shown in Fig. 3 (a)(c), and Fig. 3 (d) shows the profiles of the absorbed energy density along the line at the horizontal center of the fringes. The absorbed energy density indicates the amount of light energy absorbed by SRIG with the two beam interferometer and relates directly to the shape of the magnetic hologram pattern. In the single film (Fig. 3 (a)), the absorbed energy was large at the surface and monotonically decreased with depth as shown in Fig. 3 (d), and the width of the fringes also shrank in the deep region. However, in the finally obtained magnetic fringes, the magnetization reversed region was connected near 
the surface because of thermal diffusion from high temperature region close to the surface. This caused short fringe depth and resulted the low diffraction efficiency of the single SRIG film.

In the MPC structures, the oscillating distribution of the absorbed energy corresponding to the standing wave along thickness direction between the two Bragg mirror by means of Fabry-Pérot resonance is shown in Fig. 3 (b), (c), and (d). The multipath reflection in the MPC structure makes the distribution of absorbed energy uniform along thickness direction in the SRIG layer. Higher pair MPCs enhance the localization of light and reduce the difference in absorbed energy density between the surface and the backside of the medium. It suppressed the extreme temperature rising at the surface and form relatively small maximum temperature difference along thickness direction. This resulted the formation of deep fringes that brought the high diffraction efficiency. The effective fringe depth $d_{\mathrm{w}}$ of the single SRIG film, 1-pair MPC, and 2-pair MPC were 1.41 $\mu \mathrm{m}, 2.00 \mu \mathrm{m}$, and $2.53 \mu \mathrm{m}$, respectively. This result clearly shows that MPC structures are effective to obtain deep magnetic hologram fringes.

The resonance in MPC media also brings a large Faraday rotation angle, which is the primary role of MPCs. Fig. 5 shows the specific rotation angle of the single and MPC media. Maxima of the rotation angle appear at the designed thickness for resonance, according to Eq. (2). At an out-of-resonant thickness, for example $3.80 \mu \mathrm{m}$, the specific rotation angle of the MPC media is low, while the diffraction efficiency maintains high compared with the single film because of a deep magnetic fringe. The behavior of the thickness dependence of the specific rotation angle is close to that of the diffraction efficiency (Fig. 2) and the enhancement of rotation angle has a close relationship with the diffraction efficiency. To obtain a maximum diffraction efficiency, strict adjustment of the SRIG thickness is required. When $t_{\text {SRIG }}$ is $3.876 \mu \mathrm{m}$ at the resonant mode with $m=30$, the specific rotation angles were $2.25 \mathrm{deg} / \mu \mathrm{m}$ for the single film and $2.35 \mathrm{deg} / \mu \mathrm{m}$ for 1-pair MPC, respectively. The specific rotation angle of the 2-pair MPC was $2.68 \mathrm{deg} / \mu \mathrm{m}$ and is $24 \%$ larger than that of the single film. It contributes a large fringe rotation angle, together with an increase of the hologram depth, and results in a large diffraction efficiency.

\section{Conclusions}

We calculated the diffraction efficiencies of the MPC media with single films and MPC media to find a high performance structured medium for magnetic holograms. The calculation results showed that MPC media exhibited a high diffraction efficiency compared with single SRIG films. The 2-pair MPC medium with the SRIG thickness of $3.876 \mu \mathrm{m}$, which is the resonant thickness of Fabry-Pérot resonation, exhibited the high diffraction efficiency of $0.36 \%, 4$ times larger than that of the SRIG film. A large Faraday rotation angle of MPC structures and deep magnetic hologram fringe originated from the localization of light results in such a high diffraction efficiency of MPCs.

The diffraction efficiency of $0.36 \%$ which has been exhibited in this paper is a high value in magnetic holography, and it allows us to make a large step toward application of magnetic holography to data storage. Since the fringe rotation angle from magnetic holograms is important, the investigation for increasing the hologram depth and improving the specific rotation angle should be performed to achieve the higher diffraction efficiency.

Acknowledgements This work was supported in part by the Grants-in-Aid for Scientific Research (A) 23246060 and Grant-in-Aid for Japan Society for the Promotion of Science (JSPS) Fellows No. 25-8942.

\section{References}

1) Hans J. Coufal, Demetri Psaltis, Glenn T. Sincerbox, A. M. Glass and M. J. Cardillo: Holographic Data Storage, (Springer, New York, 2000).

2) S. Baek, H. Sakurai, P. B. Lim, A. V. Baryshev, Y. Nakamura, H. Takagi and M. Inoue: IEICE Technical Report, 111, pp.2125 (2011)

3) G. Fan, K. Pennington and J. H. Greiner: J. Appl. Phys., 40, 974 (1969).

4) H. Horimai, A. Inoue, T. Miyama, T. Fujii, S. Suzuki, R. Kusaka and T. Tokushima: J. Magn. Soc. Jpn., 8, pp.109-112 (1984) (in Japanese).

5) S. Wittekoek, T. J. A. Popma, J. M. Robertson and P. F. Bongers: Phys. Rev. B, 12, pp.2777-2788 (1975).

6) M. Inoue, R. Fujikawa, A. V. Baryshev, A. Khanikaev, P. B. Lim, H. Uchida, O. Aktsipetrov, A. Fedyanin, T. Murzina, and A. Granovsky: J. Phys. D, 39, pp.R151-R161 (2006).

7) A. M. Grishin and S. I. Khartsev: J. Magn. Soc. Jpn., 32, pp. 140-145 (2008).

8) M. Levy: J. Appl. Phys., 99, 073104 (2006).

9) N. Sagara, R. Isogai, H. Takagi, Y. Nakamura, P. B. Lim and M. Inoue, IEICE Technical Report, 113, CPM2013-90, pp.510, October 2013 (in Japanese).

Received November 29, 2013; Accepted January 17, 2014 